\title{
In vitro labeling of endothelial progenitor cells isolated from peripheral blood with superparamagnetic iron oxide nanoparticles
}

\author{
JUN-HUI SUN ${ }^{1}$, YUE-LIN ZHANG ${ }^{1}$, CHUN-HUI NIE $^{1}$, SU-PING QIAN ${ }^{2}$, \\ XIAO-BO YU ${ }^{1}$, HAI-YANG XIE ${ }^{1}$, LIN ZHOU ${ }^{1}$ and SHU-SEN ZHENG ${ }^{1}$

\begin{abstract}
${ }^{1}$ Key Laboratory of Combined Multi-Organ Transplantation, Ministry of Public Health, Key Laboratory of Organ Transplantation, and Division of Hepatobiliary and Pancreatic Surgery, Department of Surgery, First Affiliated Hospital, School of Medicine; ${ }^{2}$ Molecular Imaging Platform, Zhejiang-California International Nanosystems Institute, Zhejiang University, Hangzhou, Zhejiang, P.R. China
\end{abstract}

Received January 29, 2012; Accepted May 4, 2012

DOI: $10.3892 / \mathrm{mmr} .2012 .912$

\begin{abstract}
The transplantation of endothelial progenitor cells (EPCs) provides a novel method for the treatment of human tumors or vascular diseases. Magnetic resonance imaging (MRI) has proven to be effective in tracking transplanted stem cells by labeling the cells with superparamagnetic iron oxide (SPIO) nanoparticles. The SPIO has been used to label and track the EPCs; however, the effect of SPIO upon EPCs remains unclear on a cellular level. In the present study, EPCs were labeled with home-synthesized SPIO nanoparticles in vitro and the biological characteristics of the labeled EPCs were evaluated. The EPCs were isolated from the peripheral blood of New Zealand rabbits and cultured in fibronectin-coated culture flasks. The EPCs were labeled with home-synthesized SPIO nanoparticles at a final iron concentration of $20 \mu \mathrm{g} / \mathrm{ml}$. Labeled EPCs were confirmed with transmission electron microscopy and Prussian blue staining. The quantity of iron/cell was detected by atomic absorption spectrometry. The membranous antigens of EPCs were detected by cytofluorimetric analysis. Cell viability and proliferative capability between the labeled and unlabeled EPCs were compared. The rabbit EPCs were effectively labeled and the labeling efficiency was approximately $95 \%$. The SPIO nanoparticles were localized in the endosomal vesicles of the EPCs, which were confirmed by transmission electron microscopy. No significant differences were found in cell viability and proliferative capability between labeled and unlabeled EPCs ( $>>0.05)$. In conclusion,
\end{abstract}

Correspondence to: Professor Shu-Sen Zheng, Key Laboratory of Combined Multi-Organ Transplantation, Ministry of Public Health, Key Laboratory of Organ Transplantation, and Division of Hepatobiliary and Pancreatic Surgery, Department of Surgery, First Affiliated Hospital, School of Medicine, Zhejiang University, 79 Qingchun Road, Hangzhou, Zhejiang 310003, P.R. China E-mail: shusenzheng@zju.edu.cn

Key words: endothelial progenitor cell, superparamagnetic iron oxide nanoparticles, cell labeling rabbit peripheral blood EPCs were effectively labeled by home-synthesized SPIO nanoparticles, without influencing their main biological characteristics.

\section{Introduction}

As a potential method, cell transplantation provides a novel strategy for the therapy of incurable human diseases (1-3). Endothelial progenitor cells (EPCs) isolated from peripheral blood play a significant role in the treatment of injured blood vessels and ischemic tissues. Numerous studies have shown that EPCs exist in the peripheral blood and migrate into the damaged endothelium and neovascularization sites. They may play a role in the therapeutic procedure of repairing the endothelium and in angiogenesis $(4,5)$. Furthermore, EPCs are capable of migrating into the tumor and participating in tumor angiogenesis $(6,7)$.

In order to better understand the mechanism of EPC therapy, the in vivo monitoring of the cellular dynamics of transplanted EPCs has been proposed. A non-invasive in vivo technique that permits an evaluation of the potential migration of the transplanted EPCs would prove to be an essential tool for the treatment procedure. Many studies have indicated that magnetic resonance imaging (MRI) is effective in tracking the distribution of transplanted EPCs in vivo by labeling the cells with superparamagnetic iron oxide (SPIO) nanoparticles (8).

However, it is not very clear whether the SPIO labeling technique in EPCs is effective and safe. Additionally, the effect of SPIO upon EPCs remains unclear on a cellular level (9). The objective of the present study was to investigate whether and to what extent the labeling of EPCs with SPIO affects the main biological characteristics of EPCs.

\section{Materials and methods}

EPC culture and characterization. The present study was approved by the Animal Use and Care Committee of our institution. EPCs were generated from the peripheral blood of 5 adult New Zealand white rabbits weighing 2-2.5 kg. Blood $(20 \mathrm{ml})$ was obtained from the central ear artery of the rabbits. The fresh blood was heparinized and then diluted with 
phosphate-buffered saline (PBS), and the layer of peripheral blood mononuclear cells was selected with density centrifugation and was then resuspended in microvascular growth medium-2 (EGM-2 MV; Cambrex, Walkersville, MD, USA) supplemented with $10 \%$ fetal bovine serum (SAFC Biosciences, St. Louis, MO, USA). Before being planted in a $25-\mathrm{cm}^{3}$ culture flask, the cells were gently blown in the culture solution, adjusted to $1 \times 10^{6} / \mathrm{ml}$ of the concentration, and then grown in standard culture medium at $37^{\circ} \mathrm{C}$ with $5 \% \mathrm{CO}_{2}$. The growth and morphology of EPCs in the culture was observed every day with an inverted phase-contrast microscope (Axioscop; Zeiss Co. Ltd., Oberkochen, Germany).

In order to characterize the EPCs, the expression of membranous antigen on the cells cultured after 7 days was detected by cytofluorimetric analysis with a flow cytometer (Becton-Dickinson, San Jose, CA, USA). The primary antihuman antibodies (cross-reaction with rabbit), anti-CD31, anti-CD34 and anti-CD133 (BioLegend, San Diego, CA, USA), were employed.

EPC labeling and identification of the labeled EPCs. The SPIO $\left(\mathrm{Fe}_{3} \mathrm{O}_{4}\right)$ nanoparticles were presented by S.P. Q. (Molecular Imaging Platform, Zhejiang-California International Nanosystems Institute, Zhejiang University, Hangzhou, China). The EPCs were grown in $25-\mathrm{cm}^{3}$ flasks. After 21 days, the grown cells were transferred to the culture medium containing SPIO nanoparticles for labeling. The concentration of $20 \mu \mathrm{g} /$ $\mathrm{ml}$ iron was used for culture. The EPCs were incubated continuously for $24 \mathrm{~h}$ at $37^{\circ} \mathrm{C}$ in a $95 \%$ air $/ 5 \% \mathrm{CO}_{2}$ incubator.

The EPCs were collected by removing the free SPIO nanoparticles and washed with PBS 3 times. For the purpose of Prussian blue staining to identify the EPC profile and intracellular iron nanoparticles, the cells were continuously incubated for $20 \mathrm{~min}$ with $2 \%$ potassium ferrocyanide in $6 \%$ hydrochloric acid, and then counterstained with nuclear fast red for $3 \mathrm{~min}$.

To detect the iron concentration within the EPCs, the EPC suspension was dissolved in $37 \%$ hydrochloric acid followed by analysis with a polarized atomic absorption spectrometer (Shengyang Huaguang HG-9602A, Shengyang, China). The analysis process was repeated 3 times and the mean value was obtained. The distribution of the SPIO nanoparticles within the EPCs was shown under an electron microscope. The harvested labeled EPCs were fixed at $4^{\circ} \mathrm{C}$ in $2.5 \%$ buffered glutaraldehyde for $1 \mathrm{~h}$, followed by $1 \%$ osmium tetroxide for $2 \mathrm{~h}$. The samples of these EPCs were examined with a transmission electron microscope (H600; Hitachi, Tokyo, Japan).

Labeled EPC viability and proliferation. Cell viability and proliferative activity of SPIO-labeled and -unlabeled EPCs were evaluated and compared. All procedures were performed 3 times.

EPC viability was evaluated by trypan blue staining. The proliferative activity of the EPCs was observed under a light microscope (Axioscop; Zeiss). Additionally, tetrazolium salt (MTT) assay was performed to evaluate the toxicity and the effect of SPIO labeling upon EPC proliferation. The EPCs of passage $(\mathrm{P})_{1}$ were grown in 96 -well plates at $1 \times 10^{4}$ cells/ well. SPIO solution at a final iron concentration of $20 \mu \mathrm{g} / \mathrm{ml}$ was added into 40 wells, and the remaining 40 wells to which
SPIO was not added served as the control. The absorbance values of the unlabeled EPCs and SPIO-labeled EPCs were measured from days 1 to 5 of the culture process ( 8 wells/day). For the assay, $20 \mu \mathrm{l}$ of MTT $(5 \mathrm{mg} / \mathrm{ml}$; Fluka Co., St. Gallen, Switzerland) were added into each well and incubated at $37^{\circ} \mathrm{C}$ in $5 \% \mathrm{CO}_{2}$ for $4 \mathrm{~h}$. Dimethyl sulfoxide (DMSO; $150 \mu \mathrm{l}$; SigmaAldrich, St. Louis, MO, USA) was added and the medium was stirred for $10 \mathrm{~min}$. When the indigo crystals (formazan crystals) were dissolved evenly in the medium, the light absorption value of each well was measured with a spectrophotometer (Model 680; Bio-Rad Laboratories, Inc., Hercules, CA, USA) using a 490-nm wavelength.

Statistical analyses. Statistical analyses were performed using the SPSS ${ }^{\circledR}$ statistical package, version 11.0 (SPSS Inc., Chicago, IL, USA) for Windows ${ }^{\circledR}$. Data are presented as the means \pm standard deviation (SD). To compare the differences between the labeled and unlabeled EPCs in the various experiments, the Kruskal-Wallis rank sum test was used to calculate the difference in absorbance of MTT. A p-value (two-tailed) $<0.05$ denoted a statistically significant difference.

\section{Results}

EPC morphology and characterization. In the present study, EPCs were obtained from rabbit peripheral blood and purified by density centrifugation.

The morphology of the obtained EPCs was similar to that described in the literature (10). During culture, inverse microscopy of EPCs showed that isolated EPCs had a round shape with variable sizes (Fig. 1). After 1 week in culture, the cells became spindle-shaped, with a centrally located nucleus, and sometimes formed cluster-like colonies (Fig. 2). The cells grew and divided rapidly, and tended to touch each other. After 3 weeks, the cultured EPCs showed the typical 'cobblestone' morphology of endothelial cells when they grow in colonies (Fig. 3). When they were passed to $\mathrm{P}_{6}$, the homogeneity of the cells reached approximately $99 \%$.

The expression of surface markers, including CD34, CD31 and CD133, is typically found on EPCs as a demonstration of their immature character. The flowcytometry analyses revealed that $36.3 \pm 1.2,68.4 \pm 2.3$ and $56.0 \pm 2.5 \%$ of the cultured cells were positive for CD34, CD31 and CD133, respectively, after being cultured for 7 days (Fig. 4).

EPC labeling and identification of the labeled EPCs. The prevalence of iron content in the labeled EPCs was revealed by Prussian blue staining and transmission electron microscopy. Cells were stained with Prussian blue, and blue iron particles were found within the labeled EPCs (Fig. 5), while no blue iron particles were found in unlabeled EPCs. Microscopic cell counting post Prussian blue staining showed that the SPIO labeling rate was $>95 \%$ among all the EPCs.

The iron quantification per cell measured by an atomic absorption spectrometer was $13.6 \pm 1.8 \mathrm{pg}$. Transmission electron microscopy showed SPIO nanoparticles located in the endosomal vesicles in the cytoplasm of labeled EPCs (Fig. 6).

Labeled EPC viability and proliferative capability. Microscopic cell counting after trypan blue exclusion testing revealed 


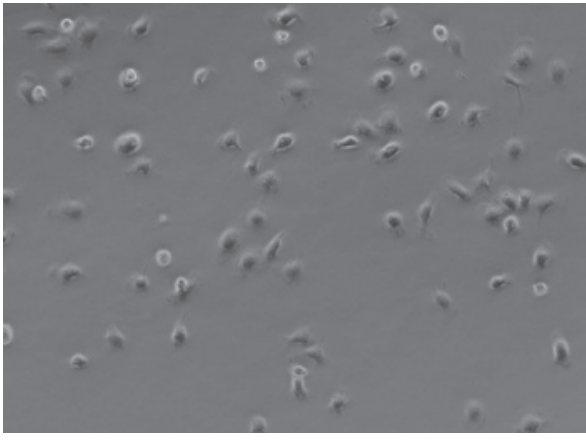

Figure 1. Under a light microscope, the endothelial progenitor cells (EPCs) after isolation and culture at day 3 had a round shape (magnification, x20).

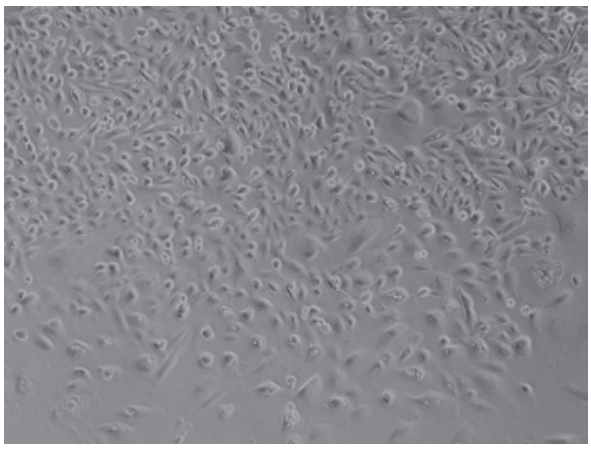

Figure 2. On the 7th day, the endothelial progenitor cells (EPCs) had a spindle shape and cell clusters had formed (magnification, x20).

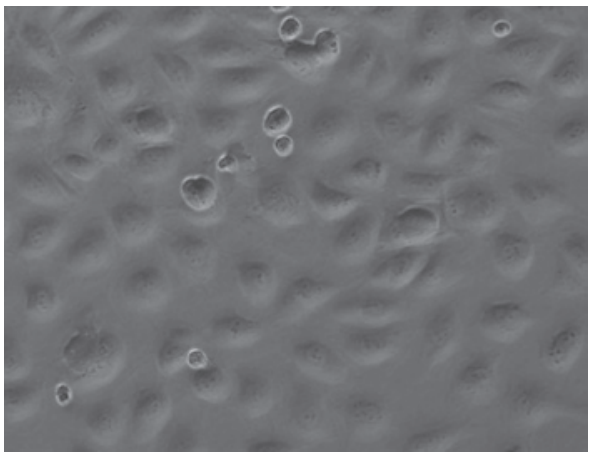

Figure 3. At day 21 after culture, the endothelial progenitor cells (EPCs) showed an endothelium-like cobblestone morphology (magnification, x100).

a mean viability of $97.5 \pm 2.1 \%$ for the SPIO-labeled EPCs. The mean viability of the unlabeled EPCs was $96.3 \pm 2.9 \%$. There were no significant differences in viability between the labeled and unlabeled EPCs $(p>0.05)$. The characteristics of the labeled EPCs, including figure, shape and nucleolus structure, did not differ from those of the unlabeled EPCs under a light microscope.

From days 1 to 5 , the absorbence of the labeled EPCs was $0.196 \pm 0.005,0.205 \pm 0.011,0.244 \pm 0.013,0.309 \pm 0.014$ and $0.363 \pm 0.022$, respectively. The absorbence of the unlabeled EPCs was $0.190 \pm 0.007,0.202 \pm 0.010,0.249 \pm 0.015$, $0.315 \pm 0.016$ and $0.377 \pm 0.019$, respectively. There were no significant differences in MTT absorbance values between the labeled and unlabeled EPCs at each time-point ( $p>0.05)$.
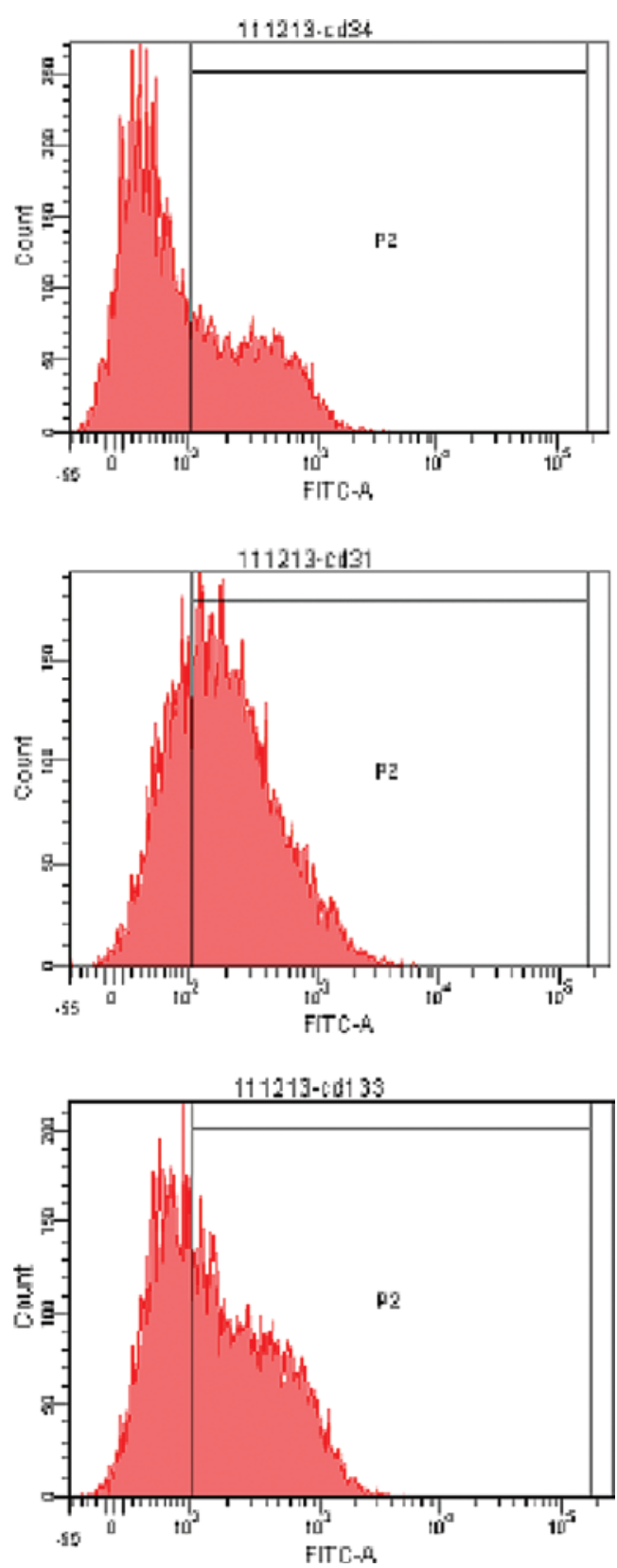

Figure 4. On the 7th day of culture, flow cytometric analysis showed that the cells were positive for endothelial progenitor cell (EPC)-related markers, CD31 (36.3\%), CD34 (68.4\%) and CD133 (56.0\%).

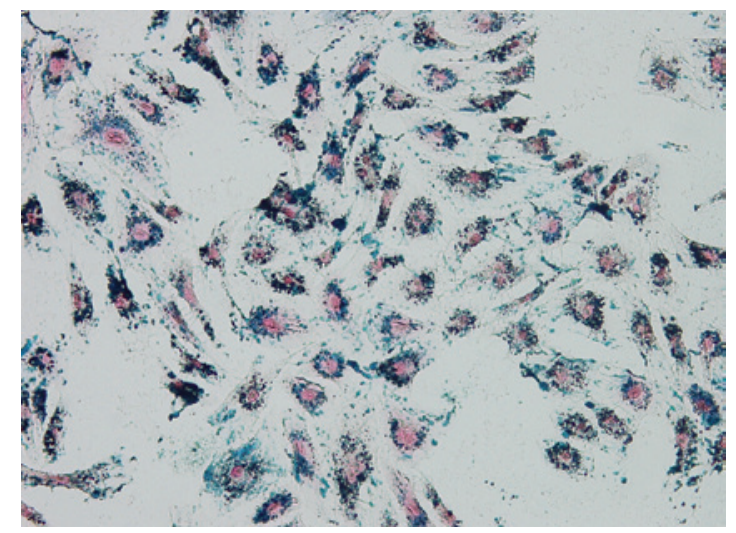

Figure 5. Prussian blue staining showing that the intracytoplasmic blue particles are clearly visible in most endothelial progenitor cells (EPCs). The labeled rate reached approximately $95 \%$ (magnification, x100). 


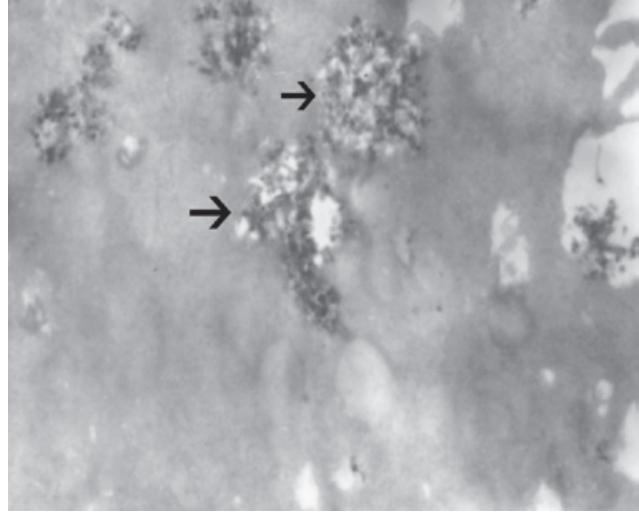

Figure 6. Transmission electron micrograph showing superparamagnetic iron oxide (SPIO) nanoparticles in black clusters (arrowhead) located in the endosomal vesicles outside the endothelial progenitor cell (EPC) nucleus (magnification, $\mathrm{x} 15,000)$

\section{Discussion}

The transplantation of stem cells is a potential strategy for the treatment of many types of human diseases due to its capability of regenerating tissues and organs. Cell transplantation has the advantages of lower cost and risk compared to organ transplantation $(11,12)$. Furthermore, autologous cell transplantation has no risk of immunological rejection. Among different types of cells, ESCs have been previously demonstrated to have better characteristics in terms of applicability for transplantation; studies have indicated that ESCs are capable of multi-directional differentiation $(13,14)$. EPCs were first isolated from human peripheral blood by Asahara et al (15). Over the past decade, the plasticity of EPCs has been intensively investigated. Many studies have demonstrated the great therapeutic potential of EPCs in tissue repair and wound healing (16-18). There are also less ethical and social controversies associated with the isolation of EPCs from bone marrow and peripheral blood, and the use of EPCs isolated from peripheral blood offers several advantages, such as easy collection and rapid in vivo and in vitro repopulation. It has been concluded by many studies that EPCs promote tissue vascular regeneration in vivo and provide potential treatments for ischemia, wound healing, vascular insufficiency and tumor inhibition (19). Moreover, EPCs play an essential role in post-natal neovascularization and maintaining angiogenesis $(18,20)$. Additionally, certain evidence suggests that EPCs have a potentially protective role in endothelial dysfunction in early atherosclerosis formation $(21,22)$.

For a better understanding of the destiny of transplanted cells following transplantation, it is essential to monitor their migration and differentiation. In order to monitor the transplanted cells, several non-invasive in vivo tracking imaging techniques, such as MRI, nuclear medicine and optical imaging, have been investigated (23-25). The MRI technique holds obvious advantages, as it has a wide variety of imaging sequences, high resolution and better soft-tissue contrast without radiation damage. The labeled cells are easily detected by MRI using a cell labeling technique with specific agents. Certain evidence implies that SPIO nanoparticles have strong penetrating capabilities among the MRI tracing agents, which makes it possible to cause signal change in MRI at a lowtracer concentration (26). A number of studies have already revealed the feasibility of in vivo tracking of the transplanted stem cells by labeling them with SPIO nanoparticles (27-29). Studies have also reported that mononuclear cells isolated from peripheral blood were tracked with MRI using colloidal superparamagnetic nanoparticles $(30,31)$. Evidently, the low efficiency of loading these particles into the cells and the cytotoxicity of these particles limit their usage as the tracing probe (32). It has been concluded by a number of studies that the SPIO nanoparticles have little toxicity and few side-effects for cell biological characteristics $(32,33)$. However, few studies have investigated the biological effect of SPIO upon labeled EPCs on a cellular level. Therefore, it is important to discover an efficient labeling method without deleterious effects on EPC viability and proliferative capability.

In the present study, we show that EPCs from peripheral blood of rabbits can be effectively labeled by home-synthesized SPIO nanoparticles. Our results demonstrated that there were no differences in cell viability and proliferative capability between the SPIO-labeled EPCs and unlabeled EPCs. Therefore, labeled home-synthesized SPIO nanoparticles have little influence on the main biological properties of EPCs. Additionally, these results are important for the application of MRI to localize and monitor the transplanted magnetically labeled EPCs by in vivo techniques.

\section{Acknowledgements}

This study was supported by the National Natural Science Foundation of China (no.30901446), the Program for Innovative Research Team of Science and Technology of Zhejiang Province (no. 2009R50038), the Foundation for Innovative Research Groups of the National Natural Science Foundation of China (no. 81121002), the Medical Health Fund of Zhejiang Province (no. 2008A053), and the Program of Chinese Medical Science of Zhejiang Province (no. 2009CB040).

\section{References}

1. Orlic D, Kajstura J, Chimenti S, et al: Mobilized bone marrow cells repair the infarcted heart, improving function and survival. Proc Natl Acad Sci USA 98: 10344-10349, 2001.

2. Sato Y, Araki H, Kato J, et al: Human mesenchymal stem cells xenografted directly to rat liver are differentiated into human hepatocytes without fusion. Blood 106: 756-763, 2005.

3. Qian $\mathrm{H}$, Yang $\mathrm{H}, \mathrm{Xu} \mathrm{W}$, et al: Bone marrow mesenchymal stem cells ameliorate rat acute renal failure by differentiation into renal tubular epithelial-like cells. Int J Mol Med 22: 325-332, 2008.

4. Murasawa S and Asahara T: Endothelial progenitor cells for vasculogenesis. Physiology 20: 36-42, 2005.

5. Schmidt-Lucke C, Rössig L, Fichtlscherer S, et al: Reduced number of circulating endothelial progenitor cells predicts future cardiovascular events: proof of concept for the clinical importance of endogenous vascular repair. Circulation 111: 2981-2987, 2005

6. Liang PH, Tian F, Lu Y, et al: Vascular endothelial growth inhibitor (VEGI; TNFSF15) inhibits bone marrow-derived endothelial progenitor cell incorporation into Lewis lung carcinoma tumors. Angiogenesis 14: 61-68, 2011.

7. George AL, Bangalore-Prakash P, Rajoria S, et al: Endothelial progenitor cell biology in disease and tissue regeneration. J Hematol Oncol 24: 24, 2011.

8. Gazeau F and Wilhelm C: Magnetic labeling, imaging and manipulation of endothelial progenitor cells using iron oxide nanoparticles. Future Med Chem 2: 397-408, 2010 
9. Yang JX, Tang WL and Wang XX: Superparamagnetic iron oxide nanoparticles may affect endothelial progenitor cell migration ability and adhesion capacity. Cytotherapy 12: 251-259, 2010.

10. Wu H, Riha GM, Yang H, et al: Differentiation and proliferation of endothelial progenitor cells from canine peripheral blood mononuclear cells. J Surg Res 126: 193-198, 2005.

11. Stutchfield BM, Forbes SJ and Wigmore SJ: Prospects for stem cell transplantation in the treatment of hepatic disease. Liver Transpl 16: 827-836, 2010.

12. Shabbir A, Zisa D, Suzuki G, et al: Heart failure therapy mediated by the trophic activities of bone marrow mesenchymal stem cells: a noninvasive therapeutic regimen. Am J Physiol Heart Circ Physiol 296: H1888-H1897, 2009.

13. Zeng $\mathrm{L}, \mathrm{Hu} \mathrm{Q}$, Wang $\mathrm{X}$, et al: Bioenergetic and functiona consequences of bone marrow-derived multipotent progenitor cell transplantation in hearts with postinfarction left ventricular remodeling. Circulation 115: 1866-1875, 2007.

14. Jickling G, Salam A, Mohammad A, et al: Circulating endothelial progenitor cells and age-related white matter changes. Stroke 40: 3191-3196, 2009.

15. Asahara T, Murohara J, Sullivan A, et al: Isolation of putative progenitor endothelial cells for angiogenesis. Science 275: 964-967, 1997.

16. Ding DC, Shyu WC, Lin SZ, et al: The role of endothelial progenitor cells in ischemic cerebral and heart diseases. Cell Transplant 16: 273-284, 2007.

17. Rotmans JI, Heyligers JM, Stroes ES, et al: Endothelial progenitor cell-seeded grafts: rash and risky. Can J Cardiol 22: $1117-1119,2006$.

18. Werner N, Kosiol S, Schieql T, et al: Circulating endothelia progenitor cells and cardiovascular outcomes. N Engl J Med 353: 999-1007, 2005

19. Moore MAS: Putting the neo into neoangiogenesis. J Clin Invest 109: 313-315, 2002.

20. Mihail H, Wolfgang E and Peter CW: Endothelial progenitor cells: mobilization, differentiation, and homing. Arterioscler Thromb Vasc Biol 23: 1185-1189, 2003.

21. Roberts N, Jahangiri M and Xu Q: Progenitor cells in vascular disease. J Cell Mol Med 9: 583-591, 2005.

22. Werner N and Nickenig G: Clinical and therapeutical implications of EPC biology in atherosclerosis. J Cell Mol Med 10: 318-332, 2006.
23. Modo M, Cash D, Mellodew K, et al: Tracking transplanted stem cell migration using bifunctional, contrast agent-enhanced, magnetic resonance imaging. Neuro Image 17: 803-811, 2002.

24. Hung SC, Deng WP, Yang WK, et al: Mesenchymal stem cell targeting of microscopic tumors and tumor stroma development monitored by noninvasive in vivo positron emission tomography imaging. Clin Cancer Res 11: 7749-7756, 2005.

25. Shichinohe H, Kuroda S, Lee JB, et al: In vivo tracking of bone marrow stromal cells transplanted into mice cerebral infarct by fluorescence optical imaging. Brain Res Brain Res Protoc 13: 166-175, 2004.

26. Weissleder R: Molecular imaging: exploring the next frontier. Radiology 212: 609-614, 1999.

27. Daldrup-Link HE, Rudelius M, Oostendorp RA, et al: targeting of hematopoietic progenitor cells with MR contrast agents. Radiology 228: 760-767, 2003.

28. Togel F, Hu Z, Weiss K, et al: Administered mesenchymal stem cells protect against ischemic acute renal failure through differentiation-independent mechanisms. Am J Physiol Renal Physiol 289: F31-F42, 2005.

29. Yocum GT, Wilson LB, Ashari P, Jordan EK, Frank JA and Arbab AS: Effect of human stem cells labeled with ferumoxides-poly-L-lysine on hematologic and biochemical measurements in rats. Radiology 235: 547-552, 2005.

30. Jendelová P, Herynek V, Urdziková L, et al: Magnetic resonance tracking of human $\mathrm{CD} 34^{+}$progenitor cells separated by means of immunomagnetic selection and transplanted into injured rat brain. Cell Transplant 14: 173-182, 2005.

31. Weber A, Pedrosa I, Kawamoto A, et al: Magnetic resonance mapping of transplanted endothelial progenitor cells for therapeutic neovascularization in ischemic heart disease. Eur J Cardiothorac Surg 26: 137-143, 2004.

32. Crabbe A, Vandeputte C, Dresselaers T, et al: Effects of MRI contrast agents on the stem cell phenotype. Cell Transplant 19: 919-936, 2010

33. Suh JS, Lee JY, Choi YS, et al: Efficient labeling of mesenchymal stem cells using cell permeable magnetic nanoparticles. Biochem Biophys Res Commun 379: 669-675, 2009. 